Proceedings of the Edinburgh Mathematical Society (2007) 50, 377-388 (C)

DOI:10.1017/S0013091505000210 Printed in the United Kingdom

\title{
BOUNDARY BEHAVIOUR OF QUOTIENTS OF MARTIN KERNELS
}

\author{
KENTARO HIRATA* \\ Department of Mathematics, Hokkaido University, Sapporo 060-0810, \\ Japan (hirata@math.sci.hokudai.ac.jp)
}

(Received 4 March 2005)

\begin{abstract}
Given two intersecting domains, we investigate the boundary behaviour of the quotient of Martin kernels of each domain. To this end, we give a characterization of minimal thinness for a difference of two subdomains in terms of Martin kernels of each domain. As a consequence of our main theorem, we obtain the boundary growth of the Martin kernel of a Lipschitz domain, which corresponds to earlier results for the boundary decay of the Green function for a Lipschitz domain investigated by Burdzy, Carroll and Gardiner.
\end{abstract}

Keywords: boundary behaviour; Martin kernel; minimal thinness

2000 Mathematics subject classification: Primary 31B05; 31B25; 31C35

\section{Introduction}

One of the aims of this paper is to examine the boundary growth of the Martin kernel of a Lipschitz domain. This is motivated by earlier work due to Burdzy, Carroll and Gardiner. We write $\mathbf{0}$ for the origin of $\mathbb{R}^{n}, n \geqslant 2$, to distinguish this from $0 \in \mathbb{R}$, and write $x=\left(x^{\prime}, x_{n}\right) \in \mathbb{R}^{n-1} \times \mathbb{R}$ and $e=\left(\mathbf{0}^{\prime}, 1\right)$. Suppose that $\phi: \mathbb{R}^{n-1} \rightarrow \mathbb{R}$ satisfies $\phi\left(\mathbf{0}^{\prime}\right)=0$ and the Lipschitz property: there is a positive constant $L$ such that

$$
\left|\phi\left(x^{\prime}\right)-\phi\left(y^{\prime}\right)\right| \leqslant L\left|x^{\prime}-y^{\prime}\right| \text { for } x^{\prime}, y^{\prime} \in \mathbb{R}^{n-1} .
$$

We put $\Omega_{\phi}=\left\{\left(x^{\prime}, x_{n}\right): x_{n}>\phi\left(x^{\prime}\right)\right\}$ and set

$$
\begin{aligned}
I^{+} & =\int_{\left\{\left|x^{\prime}\right|<1\right\}} \frac{\max \left\{\phi\left(x^{\prime}\right), 0\right\}}{\left|x^{\prime}\right|^{n}} \mathrm{~d} x^{\prime}, \\
I^{-} & =\int_{\left\{\left|x^{\prime}\right|<1\right\}} \frac{\max \left\{-\phi\left(x^{\prime}\right), 0\right\}}{\left|x^{\prime}\right|^{n}} \mathrm{~d} x^{\prime} .
\end{aligned}
$$

In $[\mathbf{2 , 3}]$, Burdzy obtained a result on the angular derivative problem for analytic functions in a Lipschitz domain. The key step was to establish a relationship between the convergence of the above integrals and the boundary behaviour of the Green function $G_{\Omega_{\phi}}$

* Present address: Faculty of Education and Human Studies, Akita University, Akita 010-8502, Japan (hirata@math.akita-u.ac.jp). 
for $\Omega_{\phi}$. Burdzy's approach was based on probabilistic methods and the minimal fine topology. Analytic proofs were given by Carroll $[\mathbf{4 , 5}]$ and Gardiner [7]. A proof based on the extremal length may be found in Sastry [13].

Theorem 1.1. Suppose that $I^{+}$and $I^{-}$are as in (1.1) and (1.2). The following statements hold.

(i) If $I^{+}<\infty$ and $I^{-}=\infty$, then

$$
\lim _{t \rightarrow 0+} \frac{G_{\Omega_{\phi}}(t e, e)}{t}=\infty .
$$

(ii) If $I^{+}=\infty$ and $I^{-}<\infty$, then

$$
\lim _{t \rightarrow 0+} \frac{G_{\Omega_{\phi}}(t e, e)}{t}=0 .
$$

(iii) If $I^{+}<\infty$ and $I^{-}<\infty$, then the limit of $G_{\Omega_{\phi}}(t e, e) / t$, as $t \rightarrow 0+$, exists and

$$
0<\lim _{t \rightarrow 0+} \frac{G_{\Omega_{\phi}}(t e, e)}{t}<\infty .
$$

In view of the boundary Harnack principle, Theorem 1.1 shows the rate of boundary decay of positive harmonic functions on $\Omega_{\phi}$ vanishing continuously on a part of the boundary of $\Omega_{\phi}$ near the origin. We are now interested in a relationship between the convergence of the integrals $I^{+}, I^{-}$and the rate of boundary growth of positive harmonic functions on $\Omega_{\phi}$ with singularity at the origin. In view of the Fatou-Naïm-Doob theorem (see [1, Theorem 9.4.6]), it is sufficient to investigate it for the Martin kernel of $\Omega_{\phi}$ with pole at the origin. (See the first paragraph of $\S 2$ for the definition of the Martin kernel.)

Theorem 1.2. Suppose that $I^{+}$and $I^{-}$are as in (1.1) and (1.2). The following statements hold.

(i) If $I^{+}<\infty$ and $I^{-}=\infty$, then

$$
\lim _{t \rightarrow 0+} t^{n-1} K_{\Omega_{\phi}}(t e, \mathbf{0})=0 .
$$

(ii) If $I^{+}=\infty$ and $I^{-}<\infty$, then

$$
\lim _{t \rightarrow 0+} t^{n-1} K_{\Omega_{\phi}}(t e, \mathbf{0})=\infty
$$

(iii) If $I^{+}<\infty$ and $I^{-}<\infty$, then the limit of $t^{n-1} K_{\Omega_{\phi}}(t e, \mathbf{0})$, as $t \rightarrow 0+$, exists and

$$
0<\lim _{t \rightarrow 0+} t^{n-1} K_{\Omega_{\phi}}(t e, \mathbf{0})<\infty .
$$

When $I^{+}=\infty$ and $I^{-}=\infty$, the limit of $t^{n-1} K_{\Omega_{\phi}}(t e, \mathbf{0})$ may take the following values: 0 , any positive and finite value, or $\infty$. 
Example 1.3. To simplify the notation, we write $\mathbb{R}_{1+}^{n-1}=\left\{x^{\prime} \in \mathbb{R}^{n-1}: x_{1} \geqslant 0\right\}$ and $\mathbb{R}_{1-}^{n-1}=\left\{x^{\prime} \in \mathbb{R}^{n-1}: x_{1} \leqslant 0\right\}$ in this example.

(i) If $\phi\left(x^{\prime}\right)$ is equal to $\frac{1}{2} x_{1}$ on $\mathbb{R}_{1+}^{n-1}$ and $x_{1}$ on $\mathbb{R}_{1-}^{n-1}$, then

$$
\lim _{t \rightarrow 0+} t^{n-1} K_{\Omega_{\phi}}(t e, \mathbf{0})=0 .
$$

(ii) If $\phi\left(x^{\prime}\right)$ is equal to $x_{1}$ on $\mathbb{R}_{1+}^{n-1}$ and $x_{1}$ on $\mathbb{R}_{1-}^{n-1}$, then the limit of $t^{n-1} K_{\Omega_{\phi}}(t e, \mathbf{0})$, as $t \rightarrow 0+$, exists and

$$
0<\lim _{t \rightarrow 0+} t^{n-1} K_{\Omega_{\phi}}(t e, \mathbf{0})<\infty
$$

(iii) If $\phi\left(x^{\prime}\right)$ is equal to $x_{1}$ on $\mathbb{R}_{1+}^{n-1}$ and $\frac{1}{2} x_{1}$ on $\mathbb{R}_{1-}^{n-1}$, then

$$
\lim _{t \rightarrow 0+} t^{n-1} K_{\Omega_{\phi}}(t e, \mathbf{0})=\infty
$$

It is easy to check that $I^{+}=\infty$ and $I^{-}=\infty$. The value of the limit in each case follows from [10, Theorems 1 and 2].

Let $\mathbb{R}_{+}^{n}=\left\{\left(x^{\prime}, x_{n}\right): x_{n}>0\right\}$. As will be explained in $\S 5$, the convergence of the integrals $I^{+}$and $I^{-}$is connected with minimal thinness of the sets $\mathbb{R}_{+}^{n} \backslash \Omega_{\phi}$ and $\Omega_{\phi} \backslash \mathbb{R}_{+}^{n}$ (see $\S 2$ for the definition of minimal thinness). Since $K_{\mathbb{R}_{+}^{n}}(t e, \mathbf{0})=t^{1-n}$, Theorem 1.2 may be interpreted as the relationship between minimal thinness of the sets $\mathbb{R}_{+}^{n} \backslash \Omega_{\phi}$, $\Omega_{\phi} \backslash \mathbb{R}_{+}^{n}$ and the boundary behaviour of the quotient of Martin kernels of $\Omega_{\phi}$ and $\mathbb{R}_{+}^{n}$. So, given two intersecting domains $\Phi$ and $\Psi$, it is valuable for us to investigate a relationship between minimal thinness of the differences $\Phi \backslash \Psi, \Psi \backslash \Phi$ and the boundary behaviour of the quotient of Martin kernels of $\Phi$ and $\Psi$ (Theorem 2.1).

\section{Statement for general domains}

Let $\Omega$ be a Greenian domain in $\mathbb{R}^{n}$ with $n \geqslant 2$. Here a Greenian domain means a domain possessing the Green function $G_{\Omega}$ for the Laplace operator. Let $x_{0}$ be a reference point in $\Omega$. The Martin kernel of $\Omega$ is defined for $(x, y) \in(\Omega \times \Omega) \backslash\left\{\left(x_{0}, x_{0}\right)\right\}$ by

$$
K_{\Omega}(x, y)=\frac{G_{\Omega}(x, y)}{G_{\Omega}\left(x_{0}, y\right)} .
$$

Now, let $\left\{y_{j}\right\}$ be a sequence in $\Omega$ with no limit point in $\Omega$. We observe by the Harnack inequality that if $j_{0}$ is sufficiently large, then $\left\{K_{\Omega}\left(\cdot, y_{j}\right)\right\}_{j} \geqslant j_{0}$ is a uniformly bounded sequence of positive harmonic functions on a given relatively compact open subset of $\Omega$. Therefore, the Harnack principle shows that there exists a subsequence $\left\{K_{\Omega}\left(\cdot, y_{j_{k}}\right)\right\}$ converging to a positive harmonic function on $\Omega$. The Martin boundary $\Delta(\Omega)$ of $\Omega$ is defined as an ideal boundary consisting of all positive harmonic functions on $\Omega$ that can be obtained as the limit of $\left\{K_{\Omega}\left(\cdot, y_{j}\right)\right\}$ for some sequence $\left\{y_{j}\right\}$ in $\Omega$ with no limit point in $\Omega$. The set $\Omega \cup \Delta(\Omega)$ (equipped with a suitable metric) is called a Martin 
compactification of $\Omega$ (see $[\mathbf{1}, \S 8.1]$ for details). Henceforth, we write $K_{\Omega}(\cdot, \xi)$ for the positive harmonic function on $\Omega$ corresponding to $\xi \in \Delta(\Omega)$. We say that $\xi \in \Delta(\Omega)$ is minimal if every positive harmonic function on $\Omega$ less than or equal to the corresponding Martin kernel $K_{\Omega}(\cdot, \xi)$ coincides with a constant multiple of $K_{\Omega}(\cdot, \xi)$. We denote the collection of all minimal Martin boundary points in $\Delta(\Omega)$ by $\Delta_{1}(\Omega)$.

The notion of minimal thinness was introduced by Naïm [12], using a regularized reduced function. Let $u$ be a positive superharmonic function on $\Omega$ and let $E$ be a subset of $\Omega$. A reduced function of $u$ relative to $E$ on $\Omega$ is defined by

$$
{ }^{\Omega} R_{u}^{E}(x)=\inf \{v(x)\},
$$

where the infimum is taken over all positive superharmonic functions $v$ on $\Omega$ such that $v \geqslant u$ on $E$. We denote the lower semicontinuous regularization of ${ }^{\Omega} R_{u}^{E}$ by ${ }^{\Omega} \hat{R}_{u}^{E}$. Observe that ${ }^{\Omega} \hat{R}_{u}^{E}$ is non-negative superharmonic on $\Omega$ and that ${ }^{\Omega} \hat{R}_{u}^{E} \leqslant u$. Let $\xi \in \Delta_{1}(\Delta)$. A set $E$ is said to be minimally thin at $\xi$ with respect to $\Omega$ if

$$
{ }^{\Omega} \hat{R}_{K_{\Omega}(\cdot, \xi)}^{E}(z)<K_{\Omega}(z, \xi) \text { for some } z \in \Omega .
$$

Minimal thinness enables us to equip the Martin compactification of $\Omega$ with the minimal fine topology. This is the collection of subsets $W$ of the Martin compactification of $\Omega$ satisfying the following conditions:

(i) $\Omega \backslash W$ is (ordinary) thin at every point of $W \cap \Omega$;

(ii) $\Omega \backslash W$ is minimally thin at every point of $W \cap \Delta_{1}(\Omega)$.

See $[\mathbf{1}$, Chapter 7$]$ for details on ordinary thinness. Let $U$ be a minimal fine neighbourhood of $\xi \in \Delta_{1}(\Omega)$. We say that a function $f$ on $U$ has minimal fine limit $l$ at $\xi$ with respect to $\Omega$ if there is a subset $E$ of $\Omega$, minimally thin at $\xi$ with respect to $\Omega$, such that $f(x) \rightarrow l$ as $x \rightarrow \xi$ along $U \backslash E$, and then we write

$$
\min _{\Omega} \lim _{x \rightarrow \xi} f(x)=l .
$$

We note from the definition that a function is not necessarily defined on the whole of a domain when we consider the minimal fine limit.

Theorem 2.1 (main result). Let $\Phi$ and $\Psi$ be Greenian domains in $\mathbb{R}^{n}$ such that $\Phi \cap \Psi$ is a non-empty domain. Suppose that $\xi \in \Delta_{1}(\Phi)$ is in the closure of $\Phi \cap \Psi$ in the Martin compactification of $\Phi$, and that $\zeta \in \Delta_{1}(\Psi)$ is in the closure of $\Phi \cap \Psi$ in the Martin compactification of $\Psi$. If $\Phi \backslash \Psi$ is minimally thin at $\xi$ with respect to $\Phi$, then $K_{\Psi}(\cdot, \zeta) / K_{\Phi}(\cdot, \xi)$ has a finite minimal fine limit at $\xi$ with respect to $\Phi$. Furthermore, the following statements hold.

(i) If $\Psi \backslash \Phi$ is not minimally thin at $\zeta$ with respect to $\Psi$, then

$$
\operatorname{mf}_{\Phi} \lim _{x \rightarrow \xi} \frac{K_{\Psi}(x, \zeta)}{K_{\Phi}(x, \xi)}=0
$$




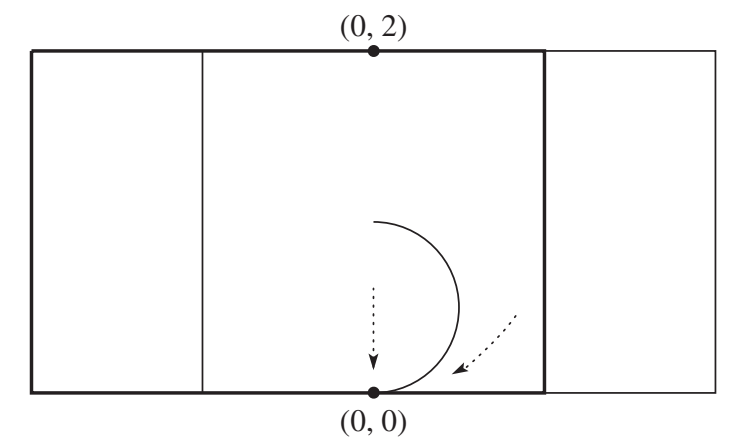

Figure 1. Condition (2.1).

(ii) If $\Psi \backslash \Phi$ is minimally thin at $\zeta$ with respect to $\Psi$, where $\zeta$ is the point such that

$$
K_{\Psi}(\cdot, \zeta)-{ }^{\Psi} R_{K_{\Psi}(\cdot, \zeta)}^{\Psi \backslash \Phi}=\alpha\left(K_{\Phi}(\cdot, \xi)-{ }^{\Phi} R_{K_{\Phi}(\cdot, \xi)}^{\Phi \backslash \Psi}\right) \quad \text { on } \Phi \cap \Psi
$$

for some positive constant $\alpha$, then

$$
0<\operatorname{mf}_{\Phi} \lim _{x \rightarrow \xi} \frac{K_{\Psi}(x, \zeta)}{K_{\Phi}(x, \xi)}<\infty
$$

(iii) If $\Psi \backslash \Phi$ is minimally thin at $\zeta$ with respect to $\Psi$, where $\zeta$ is a point such that (2.1) is not satisfied, then

$$
\operatorname{mf}_{\Phi} \lim _{x \rightarrow \xi} \frac{K_{\Psi}(x, \zeta)}{K_{\Phi}(x, \xi)}=0
$$

We explain condition (2.1) using the following example.

Example 2.2. Let

$$
\begin{aligned}
& \Phi=\left\{\left(x_{1}, x_{2}\right) \in \mathbb{R}^{2}:-2<x_{1}<1,0<x_{2}<2\right\}, \\
& \Psi=\left\{\left(x_{1}, x_{2}\right) \in \mathbb{R}^{2}:-1<x_{1}<2,0<x_{2}<2\right\} \backslash E,
\end{aligned}
$$

where

$$
E=\left\{\left(x_{1}, x_{2}\right) \in \mathbb{R}^{2}:\left|x_{1}\right|^{2}+\left|x_{2}-\frac{1}{2}\right|^{2}=\frac{1}{4}, x_{1}>0\right\} .
$$

See Figure 1. Observe that the Martin boundary of $\Phi$ is homeomorphic to its Euclidean boundary and all points are minimal, and that there are two minimal Martin boundary points, say $\zeta_{1}, \zeta_{2}$, over $(0,0)$ with respect to $\Psi$. Suppose that $K_{\Psi}\left(\cdot, \zeta_{1}\right)$ is the limit of $K_{\Psi}(\cdot, y)$ as $y \rightarrow(0,0)$ along $\left\{\left(0, x_{2}\right): 0<x_{2}<1\right\}$, and that $K_{\Psi}\left(\cdot, \zeta_{2}\right)$ is the limit of $K_{\Psi}(\cdot, y)$ as $y \rightarrow(0,0)$ along $\left\{\left(x_{1}, x_{2}\right):\left|x_{1}\right|^{2}+\left|x_{2}-1\right|^{2}=1, x_{1}>0\right\}$. Also, let $\zeta_{3}=(0,2)$. Then $\Phi \backslash \Psi$ is minimally thin at $(0,0)$ with respect to $\Phi$, and $\Psi \backslash \Phi$ is minimally thin at $\zeta_{j}, j=1,2,3$, with respect to $\Psi$. It is not difficult to see that $\zeta_{1}$ satisfies condition (2.1), but $\zeta_{2}$ and $\zeta_{3}$ do not. 
For Lipschitz domains $\Phi$ and $\Psi$, Theorem 2.1 can be restated as the corollary below. We note from [9] that each Euclidean boundary point of a Lipschitz domain corresponds in a natural way to a unique Martin boundary point and it is minimal. So, Martin boundary points and Euclidean boundary points may be identified in this setting. Let $\Omega$ be a Lipschitz domain in $\mathbb{R}^{n}$ and let $c>1$. We define a non-tangential region at $y \in \partial \Omega$ (the Euclidean boundary of $\Omega$ ) by

$$
\Gamma_{c}(y)=\{x \in \Omega:|x-y|<c \operatorname{dist}(x, \partial \Omega)\} .
$$

Note that this region is non-empty once $c$ is sufficiently large. We say that a function $f$ on $\Omega$ has non-tangential limit $l$ at $y$ if, for each $c$ sufficiently large, $f(x)$ has limit $l$ as $x \rightarrow y$ along $\Gamma_{c}(y)$. Then we write

$$
\mathrm{nt}_{\Omega} \lim _{x \rightarrow y} f(x)=l .
$$

Corollary 2.3. Suppose that $\Phi$ and $\Psi$ are Lipschitz domains in $\mathbb{R}^{n}$ such that $\Phi \cap \Psi$ is also a Lipschitz domain. Let $y \in \partial \Phi \cap \partial \Psi$, and suppose that $\Phi \backslash \Psi$ is minimally thin at $y$ with respect to $\Phi$. The following statements hold.

(i) If $\Psi \backslash \Phi$ is not minimally thin at $y$ with respect to $\Psi$, then

$$
\operatorname{nt}_{\Phi \cap \Psi}-\lim _{x \rightarrow y} \frac{K_{\Psi}(x, y)}{K_{\Phi}(x, y)}=0 .
$$

(ii) If $\Psi \backslash \Phi$ is minimally thin at $y$ with respect to $\Psi$, then the non-tangential limit of $K_{\Psi}(\cdot, y) / K_{\Phi}(\cdot, y)$ at $y$ with respect to $\Phi \cap \Psi$ exists and

$$
0<\operatorname{nt}_{\Phi \cap \Psi}-\lim _{x \rightarrow y} \frac{K_{\Psi}(x, y)}{K_{\Phi}(x, y)}<\infty .
$$

Remark 2.4. If $\Phi \backslash \Psi$ is not minimally thin at $y$ with respect to $\Phi$ and $\Psi \backslash \Phi$ is not minimally thin at $y$ with respect to $\Psi$, then the non-tangential limit of $K_{\Psi}(\cdot, y) / K_{\Phi}(\cdot, y)$ may take the following values: 0 , any positive and finite value, or $\infty$ (see Example 1.3).

\section{Characterization of minimal thinness for a difference of two subdomains}

Naïm [12, Théorème 11] gave a characterization of minimal thinness for a difference of two subdomains in terms of Green functions for each domain, which played an important role in the proof of Theorem 1.1. In order to prove Theorem 2.1, we need a new characterization of minimal thinness for a difference.

Lemma 3.1. Let $\Omega$ be a Greenian domain in $\mathbb{R}^{n}$ and let $D$ be a subdomain of $\Omega$. Suppose that $\xi \in \Delta_{1}(\Omega)$ is in the closure of $D$ in the Martin compactification of $\Omega$. The following statements are equivalent:

(i) $\Omega \backslash D$ is minimally thin at $\xi$ with respect to $\Omega$;

(ii) there exists $\eta \in \Delta_{1}(D)$ such that

$$
\operatorname{mf}_{D} \lim _{x \rightarrow \eta} \frac{K_{\Omega}(x, \xi)}{K_{D}(x, \eta)}>0 .
$$


Furthermore, the point $\eta \in \Delta_{1}(D)$ in (ii) is uniquely determined and the corresponding Martin kernel is represented as

$$
K_{D}(\cdot, \eta)=\alpha\left(K_{\Omega}(\cdot, \xi)-{ }^{\Omega} R_{K_{\Omega}(\cdot, \xi)}^{\Omega \backslash D}\right) \quad \text { on } D
$$

for some positive constant $\alpha$.

Remark 3.2. We note that the minimal fine limit in (3.1) always exists and that it satisfies

$$
\operatorname{mf}_{D}-\lim _{x \rightarrow \eta} \frac{K_{\Omega}(x, \xi)}{K_{D}(x, \eta)}=\mu_{K_{\Omega}(\cdot, \xi)}^{D}(\{\eta\})=\inf _{x \in D} \frac{K_{\Omega}(x, \xi)}{K_{D}(x, \eta)}=\liminf _{x \rightarrow \eta} \frac{K_{\Omega}(x, \xi)}{K_{D}(x, \eta)}<\infty,
$$

where $\mu_{K_{\Omega}(\cdot, \xi)}^{D}$ is the measure on $\Delta(D)$ associated with $K_{\Omega}(\cdot, \xi)$ in the Martin representation (see [1, Theorems 9.2.6 and 9.3.3]. Thus, minimal thinness of $\Omega \backslash D$ can also be characterized in terms of any of the quantities in (3.2) instead of the minimal fine limit.

For the proof of Lemma 3.1, we need the following lemmas. Lemma 3.3 can be deduced from [1, Theorems 9.2.6 and 9.3.3]. Lemma 3.4 is due to Naïm [12, Théorème 15] (cf. [1, Theorem 9.5.5]).

Lemma 3.3. Let $E$ be a subset of a Greenian domain $\Omega$ in $\mathbb{R}^{n}$ and let $\xi \in \Delta_{1}(\Omega)$. The following statements are equivalent:

(i) $E$ is minimally thin at $\xi$ with respect to $\Omega$;

(ii) there exists a positive superharmonic function $u$ on $\Omega$ such that

$$
\inf _{x \in \Omega} \frac{u(x)}{K_{\Omega}(x, \xi)}<\inf _{x \in E} \frac{u(x)}{K_{\Omega}(x, \xi)} .
$$

Lemma 3.4. Let $\Omega$ be a Greenian domain in $\mathbb{R}^{n}$ and let $D$ be a subdomain of $\Omega$. Suppose that $\xi \in \Delta_{1}(\Omega)$ is in the closure of $D$ in the Martin compactification of $\Omega$. Assume that $\Omega \backslash D$ is minimally thin at $\xi$ with respect to $\Omega$, and let $\eta \in \Delta_{1}(D)$ be the point such that

$$
K_{D}(\cdot, \eta)=\alpha\left(K_{\Omega}(\cdot, \xi)-{ }^{\Omega} R_{K_{\Omega}(\cdot, \xi)}^{\Omega \backslash D}\right) \quad \text { on } D
$$

for some positive constant $\alpha$. The following statements for a subset $E$ of $D$ are equivalent:

(i) $E$ is minimally thin at $\eta$ with respect to $D$;

(ii) $E$ is minimally thin at $\xi$ with respect to $\Omega$.

We say that a property holds quasi-everywhere if it holds apart from a polar set. The following lemma is elementary. For the convenience of the reader, we give a proof.

Lemma 3.5. Let $D$ be a Greenian domain in $\mathbb{R}^{n}$ and let $\zeta \in \Delta_{1}(D)$. Then $K_{D}(\cdot, \zeta)$ vanishes quasi-everywhere on $\partial D$. 
Proof. Let $V$ be a Martin topology (closed) neighbourhood of $\zeta$ with respect to $D$. Then $V \cap D$ is not minimally thin at $\zeta$ with respect to $D$. Therefore, from $[\mathbf{1}$, Theorem 6.9.1], we have

$$
K_{D}(x, \zeta)={ }^{D} R_{K_{D}(\cdot, \zeta)}^{V \cap D}(x)=H_{K_{D}(\cdot, \zeta) \mathcal{X}_{\partial V \cap D}}^{D \backslash V}(x) \quad \text { for } x \in D \backslash V,
$$

where $H_{K_{D}(\cdot, \zeta) \mathcal{X}_{\partial V \cap D}}^{D \backslash V}$ denotes the Perron-Wiener-Brelot solution of the Dirichlet problem in $D \backslash V$ with the boundary function $K_{D}(\cdot, \zeta)$ on $\partial(V \cap D) \cap D$ and 0 on $\partial D$. Since $V$ is arbitrary, we obtain the lemma.

Let $\Omega$ be a domain in $\mathbb{R}^{n}$ and let $D$ be a subdomain of $\Omega$. If $h$ is a positive harmonic function on $D$ that vanishes quasi-everywhere on $\partial D \cap \Omega$ and is bounded near each point of $\partial D \cap \Omega$, then we see from [1, Theorem 5.2.1] that $h$ has a subharmonic extension $h^{*}$ to $\Omega$ that is valued 0 quasi-everywhere on $\partial D \cap \Omega$ and everywhere on $\Omega \backslash \bar{D}$. In what follows, we use an asterisk, as in $h^{*}$, to denote such a subharmonic extension.

Let us prove Lemma 3.1 .

Proof of Lemma 3.1. By [12, Théorème 12] (cf. [1, Theorem 9.5.5]), we can easily show that (i) implies (ii). In fact,

$$
f:=K_{\Omega}(\cdot, \xi)-{ }^{\Omega} R_{K_{\Omega}(\cdot, \xi)}^{\Omega \backslash D}
$$

is a minimal harmonic function on $D$, and so there exists $\eta \in \Delta_{1}(D)$ such that $K_{D}(\cdot, \eta)=$ $f / f\left(x_{0}\right)$ on $D$. Hence, we obtain

$$
\inf _{x \in D} \frac{K_{\Omega}(x, \xi)}{K_{D}(x, \eta)} \geqslant f\left(x_{0}\right)>0,
$$

and thus (3.1) follows from (3.2).

We next show that (ii) implies (i). We may assume that $\Omega \backslash D$ is non-polar. Let $\eta \in \Delta_{1}(D)$ be a point such that

$$
\alpha:=\operatorname{mf}_{D} \lim _{x \rightarrow \eta} \frac{K_{\Omega}(x, \xi)}{K_{D}(x, \eta)}>0 .
$$

By (3.2), we have $K_{D}(\cdot, \eta) \leqslant \alpha^{-1} K_{\Omega}(\cdot, \xi)$ on $D$. This shows that $K_{D}(\cdot, \eta)$ is bounded near each point of $\partial D \cap \Omega$. Also, $K_{D}(\cdot, \eta)$ vanishes quasi-everywhere on $\partial D \cap \Omega$ by Lemma 3.5. Thus, $K_{D}^{*}(\cdot, \eta)$ is well defined as a subharmonic function on $\Omega$ and is dominated by $\alpha^{-1} K_{\Omega}(\cdot, \xi)$ on $\Omega$. Let $u=\alpha^{-1} K_{\Omega}(\cdot, \xi)-K_{D}^{*}(\cdot, \eta)$. Then $u$ is superharmonic on $\Omega$. Since $\Omega \backslash D$ is non-polar, there is a point in $\Omega \backslash D$ at which $u$ is positive. Therefore, the minimum principle yields that $u$ is positive on $\Omega$. Also, we find that

$$
\begin{gathered}
\inf _{x \in \Omega} \frac{u(x)}{K_{\Omega}(x, \xi)}=\alpha^{-1}-\sup _{x \in D} \frac{K_{D}(x, \eta)}{K_{\Omega}(x, \xi)}<\alpha^{-1}, \\
\inf _{x \in \Omega \backslash(D \cup F)} \frac{u(x)}{K_{\Omega}(x, \xi)}=\alpha^{-1}-\sup _{x \in \Omega \backslash(D \cup F)} \frac{K_{D}^{*}(x, \eta)}{K_{\Omega}(x, \xi)}=\alpha^{-1},
\end{gathered}
$$


where $F$ is a polar set in $\partial D \cap \Omega$ such that $K_{D}^{*}(\cdot, \eta)>0$ on $F$. Hence, it follows from Lemma 3.3 that $\Omega \backslash(D \cup F)$ is minimally thin at $\xi$ with respect to $\Omega$, and so is $\Omega \backslash D$.

We finally show the uniqueness of $\eta \in \Delta_{1}(D)$. We suppose to the contrary that there exists $\zeta \in \Delta_{1}(D)$ such that $K_{D}(\cdot, \zeta) \leqslant \beta K_{\Omega}(\cdot, \xi)$ on $D$ and $K_{D}(\cdot, \zeta)$ is different from

$$
K_{D}(\cdot, \eta):=\gamma\left(K_{\Omega}(\cdot, \xi)-{ }^{\Omega} R_{K_{\Omega}(\cdot, \xi)}^{\Omega \backslash D}\right)
$$

where $\beta$ and $\gamma$ are some positive constants. We may assume that $\beta$ is the smallest number satisfying $K_{D}(\cdot, \zeta) \leqslant \beta K_{\Omega}(\cdot, \xi)$ on $D$. Since $\xi \in \Delta_{1}(\Omega)$, it follows that $\beta K_{\Omega}(\cdot, \xi)$ is the least harmonic majorant of $K_{D}^{*}(\cdot, \zeta)$ on $\Omega$. Let $W$ be a Martin topology neighbourhood of $\zeta$ with respect to $D$ such that $\eta$ is apart from $W$. Then $W \cap D$ is minimally thin at $\eta$ with respect to $D$. Thus, minimal thinness of $\Omega \backslash D$ at $\xi$ with respect to $\Omega$, together with Lemma 3.4, yields that $W \cap D$ is minimally thin at $\xi$ with respect to $\Omega$.

On the other hand, since $W \cap D$ is not minimally thin at $\zeta$ with respect to $D$, we have

$$
K_{D}(\cdot, \zeta)={ }^{D} \hat{R}_{K_{D}(\cdot, \zeta)}^{W \cap D} \leqslant \beta^{D} \hat{R}_{K_{\Omega}(\cdot, \xi)}^{W \cap D} \leqslant \beta^{\Omega} \hat{R}_{K_{\Omega}(\cdot, \xi)}^{W \cap D} \quad \text { on } D .
$$

Since $\beta K_{\Omega}(\cdot, \xi)$ is the least one among superharmonic functions $u$ on $\Omega$ satisfying $K_{D}^{*}(\cdot, \zeta) \leqslant u$ on $\Omega$, we have ${ }^{\Omega} \hat{R}_{K_{\Omega}(\cdot, \xi)}^{W \cap D}=K_{\Omega}(\cdot, \xi)$ on $\Omega$, so that $W \cap D$ is not minimally thin at $\xi$ with respect to $\Omega$. Thus, we obtain a contradiction, and hence the uniqueness of $\eta \in \Delta_{1}(D)$ is established. The proof of Lemma 3.1 is complete.

\section{Proofs of Theorem 2.1 and Corollary 2.3}

We give proofs of Theorem 2.1 and Corollary 2.3.

Proof of Theorem 2.1. In order to prove the first assertion, we assume that $\Phi \backslash(\Phi \cap \Psi)$ is minimally thin at $\xi$ with respect to $\Phi$. Let $\eta \in \Delta_{1}(\Phi \cap \Psi)$ be the point such that

$$
K_{\Phi \cap \Psi}(\cdot, \eta)=\alpha\left(K_{\Phi}(\cdot, \xi)-{ }^{\Phi} R_{K_{\Phi}(\cdot, \xi)}^{\Phi \backslash \Psi}\right)
$$

on $\Phi \cap \Psi$ for some positive constant $\alpha$. Then, by Lemma 3.1 with $D:=\Phi \cap \Psi$ and $\Omega:=\Phi$ and Remark 3.2, we find that the minimal fine limit of $K_{\Phi}(\cdot, \xi) / K_{\Phi \cap \Psi}(\cdot, \eta)$ at $\eta$ with respect to $\Phi \cap \Psi$ exists and

$$
0<\operatorname{mf}_{\Phi \cap \Psi x \rightarrow \eta} \frac{K_{\Phi}(x, \xi)}{K_{\Phi \cap \Psi}(x, \eta)}<\infty
$$

It also follows from $\left[\mathbf{1}\right.$, Theorem 9.3.3] that $K_{\Psi}(\cdot, \zeta) / K_{\Phi \cap \Psi}(\cdot, \eta)$ has a finite minimal fine limit at $\eta$ with respect to $\Phi \cap \Psi$. The minimal thinness of $\Phi \backslash(\Phi \cap \Psi)$ at $\xi$ with respect to $\Phi$, together with Lemma 3.4 with $D:=\Phi \cap \Psi$ and $\Omega:=\Phi$, allows us to conclude that $K_{\Psi}(\cdot, \zeta) / K_{\Phi}(\cdot, \xi)$ has a finite minimal fine limit at $\xi$ with respect to $\Phi$. 
To prove (i), we assume in addition that $\Psi \backslash(\Phi \cap \Psi)$ is not minimally thin at $\zeta$ with respect to $\Psi$. Then Lemma 3.1 with $D:=\Phi \cap \Psi$ and $\Omega:=\Psi$ shows that, for any $\eta \in \Delta_{1}(\Phi \cap \Psi)$, the minimal fine limit in (3.1) is zero. Therefore, we have that

$$
\operatorname{mf}_{\Phi \cap \Psi x \rightarrow \eta} \frac{K_{\Psi}(x, \zeta)}{K_{\Phi \cap \Psi}(x, \eta)}=0 .
$$

Hence, (i) follows from (4.1) and Lemma 3.4 with $D:=\Phi \cap \Psi$ and $\Omega:=\Phi$.

To prove (ii), we assume in addition that $\Psi \backslash(\Phi \cap \Psi)$ is minimally thin at $\zeta$ with respect to $\Psi$, where $\zeta$ is the point in $\Delta_{1}(\Psi)$ such that $(2.1)$ is satisfied. We note from (2.1) that $K_{\Phi \cap \Psi}(\cdot, \eta)$ is also written as $\beta\left(K_{\Psi}(\cdot, \zeta)-{ }^{\Psi} R_{K_{\Psi}(\cdot, \zeta)}^{\Psi \backslash \Phi}\right)$ on $\Phi \cap \Psi$ for some positive constant $\beta$. Then, by Lemma 3.1 with $D:=\Phi \cap \Psi$ and $\Omega:=\Psi$ and Remark 3.2, we find that the minimal fine limit of $K_{\Psi}(\cdot, \zeta) / K_{\Phi \cap \Psi}(\cdot, \eta)$ at $\eta$ with respect to $\Phi \cap \Psi$ exists and

$$
0<\operatorname{mf}_{\Phi \cap \Psi}-\lim _{x \rightarrow \eta} \frac{K_{\Psi}(x, \zeta)}{K_{\Phi \cap \Psi}(x, \eta)}<\infty .
$$

Therefore, (ii) follows from (4.1) and Lemma 3.4 with $D:=\Phi \cap \Psi$ and $\Omega:=\Phi$.

To prove (iii), we assume in addition that $\Psi \backslash(\Phi \cap \Psi)$ is minimally thin at $\zeta$ with respect to $\Psi$, where $\zeta$ is a point in $\Delta_{1}(\Psi)$ such that (2.1) is not satisfied. Then the normalization $K_{\Phi \cap \Psi}(\cdot, \omega)$ of $K_{\Psi}(\cdot, \zeta)-{ }^{\Psi} R_{K_{\Psi} \backslash(\cdot, \zeta)} \backslash$ at a reference point is a minimal Martin kernel of $\Phi \cap \Psi$, but is different from $K_{\Phi \cap \Psi}(\cdot, \eta)$. We note from the uniqueness in Lemma 3.1 that for only $\omega \in \Delta_{1}(\Phi \cap \Psi), K_{\Psi}(\cdot, \zeta) / K_{\Phi \cap \Psi}(\cdot, \omega)$ has a positive minimal fine limit at $\omega$ with respect to $\Phi \cap \Psi$. Therefore, we have

$$
\operatorname{mf}_{\Phi \cap \Psi}-\lim _{x \rightarrow \eta} \frac{K_{\Psi}(x, \zeta)}{K_{\Phi \cap \Psi}(x, \eta)}=0 .
$$

Hence, (iii) follows from (4.1) and Lemma 3.4 with $D:=\Phi \cap \Psi$ and $\Omega:=\Phi$. Thus, Theorem 2.1 is established.

Proof of Corollary 2.3. Let $y \in \partial \Phi \cap \partial \Psi$. We first show (i). By Theorem 2.1 (i) and Lemma 3.4, we find that $K_{\Psi}(\cdot, y) / K_{\Phi}(\cdot, y)$ has minimal fine limit 0 at $y$ with respect to $\Phi \cap \Psi$. Since the non-tangential region $\Gamma_{c}(y)$ is not minimally thin at $y$ with respect to $\Phi \cap \Psi$ (see $[\mathbf{9}, \S 5]$ ), the existence of the minimal fine limit of $K_{\Psi}(\cdot, y) / K_{\Phi}(\cdot, y)$ with respect to $\Phi \cap \Psi$ implies the existence of the non-tangential limit with respect to $\Phi \cap \Psi$, and the both values coincide. Hence, (i) follows.

We next show (ii). We observe that $K_{\Phi}(\cdot, y)$ and $K_{\Psi}(\cdot, y)$ satisfy $(2.1)$ on $\Phi \cap \Psi$, since

$$
K_{\Phi}(\cdot, y)-{ }^{\Phi} R_{K_{\Phi}(\cdot, y)}^{\Phi \backslash \Psi} \quad \text { and } \quad K_{\Psi}(\cdot, y)-{ }^{\Psi} R_{K_{\Psi}(\cdot, y)}^{\Psi \backslash \Phi}
$$

are minimal harmonic functions on $\Phi \cap \Psi$ with a pole at $y$. Therefore, (ii) follows from Theorem 2.1 (ii). 


\section{Proof of Theorem 1.2}

In order to prove Theorem 1.2, we collect lemmas on relationships between the convergence of the integrals $I^{+}, I^{-}$in (1.1), (1.2) and minimal thinness of the differences $\Omega_{\phi} \backslash \mathbb{R}_{+}^{n}, \mathbb{R}_{+}^{n} \backslash \Omega_{\phi}$. (See $[\mathbf{7}$, Lemma 1 and proof of Theorem 1] for Lemma 5.1 and [6, Theorem 4.2] for Lemma 5.2.)

Lemma 5.1. The following statements hold.

(i) $I^{+}<\infty$ if and only if $\mathbb{R}_{+}^{n} \backslash \Omega_{\phi}$ is minimally thin at $\mathbf{0}$ with respect to $\mathbb{R}_{+}^{n}$.

(ii) If $I^{+}<\infty$ and $I^{-}=\infty$, then $\Omega_{\phi} \backslash \mathbb{R}_{+}^{n}$ is not minimally thin at $\mathbf{0}$ with respect to $\Omega_{\phi}$.

Lemma 5.2. Let $\Omega$ be a Greenian domain in $\mathbb{R}^{n}$ containing $\mathbb{R}_{+}^{n}$. Suppose that $\Omega$ has a unique Martin boundary point at infinity and it is minimal. If $\Omega \backslash \overline{\mathbb{R}}_{+}^{n}$ is minimally thin at $\infty$ with respect to $\mathbb{R}_{-}^{n}:=\left\{\left(x^{\prime}, x_{n}\right): x_{n}<0\right\}$, then $\Omega \backslash \mathbb{R}_{+}^{n}$ is minimally thin at $\infty$ with respect to $\Omega$.

Lemma 5.3. If $I^{-}<\infty$, then $\Omega_{\phi} \backslash \mathbb{R}_{+}^{n}$ is minimally thin at $\mathbf{0}$ with respect to $\Omega_{\phi} \cup \mathbb{R}_{+}^{n}$.

Proof. By Lemma 5.1, we see that $\Omega_{\phi} \backslash \overline{\mathbb{R}}_{+}^{n}$ is minimally thin at $\mathbf{0}$ with respect to $\mathbb{R}_{-}^{n}$. Since minimal thinness is invariant under the inversion with respect to the unit sphere, it follows from Lemma 5.2 that $\Omega_{\phi} \backslash \mathbb{R}_{+}^{n}$ is minimally thin at $\mathbf{0}$ with respect to $\Omega_{\phi} \cup \mathbb{R}_{+}^{n}$.

Lemma 5.4. If $I^{+}<\infty$ and $I^{-}<\infty$, then $\Omega_{\phi} \backslash \mathbb{R}_{+}^{n}$ is minimally thin at $\mathbf{0}$ with respect to $\Omega_{\phi}$.

Proof. We note from Lemma 5.3 that $\left(\Omega_{\phi} \cup \mathbb{R}_{+}^{n}\right) \backslash \mathbb{R}_{+}^{n}$ is minimally thin at $\mathbf{0}$ with respect to $\Omega_{\phi} \cup \mathbb{R}_{+}^{n}$. Therefore, we see from Lemmas 3.4 and 5.1 that $\left(\Omega_{\phi} \cup \mathbb{R}_{+}^{n}\right) \backslash \Omega_{\phi}$ is minimally thin at $\mathbf{0}$ with respect to $\Omega_{\phi} \cup \mathbb{R}_{+}^{n}$. Applying Lemma 3.4 again, we obtain Lemma 5.4.

Let us now prove Theorem 1.2.

Proof of Theorem 1.2. We can easily obtain (i) and (ii) from Corollary 2.3 with $\Phi:=\mathbb{R}_{+}^{n}$ and $\Psi:=\Omega_{\phi}$ and Lemmas 5.1 and 5.4. We show (2). Since $\left(\Omega_{\phi} \cup \mathbb{R}_{+}^{n}\right) \backslash \mathbb{R}_{+}^{n}$ is minimally thin at $\mathbf{0}$ with respect to $\Omega_{\phi} \cup \mathbb{R}_{+}^{n}$ by Lemma 5.3 , it follows, by Lemma 3.1 with $D:=\mathbb{R}_{+}^{n}$ and $\Omega:=\Omega_{\phi} \cup \mathbb{R}_{+}^{n}$, that $K_{\Omega_{\phi} \cup \mathbb{R}_{+}^{n}}(\cdot, \mathbf{0}) / K_{\mathbb{R}_{+}^{n}}(\cdot, \mathbf{0})$ has a positive minimal fine limit at $\mathbf{0}$ with respect to $\mathbb{R}_{+}^{n}$. Therefore, $t^{n-1} K_{\Omega_{\phi} \cup \mathbb{R}_{+}^{n}}(t e, \mathbf{0})$ has a positive limit as $t \rightarrow 0+$. Also, it follows from Lemmas 3.4 and 5.1 that $\left(\Omega_{\phi} \cup \mathbb{R}_{+}^{n}\right) \backslash \Omega_{\phi}$ is not minimally thin at $\mathbf{0}$ with respect to $\Omega_{\phi} \cup \mathbb{R}_{+}^{n}$. Therefore, by Lemma 3.1 with $D:=\Omega_{\phi}$ and $\Omega:=\Omega_{\phi} \cup \mathbb{R}_{+}^{n}$, we see that $K_{\Omega_{\phi} \cup \mathbb{R}_{+}^{n}}(\cdot, \mathbf{0}) / K_{\Omega_{\phi}}(\cdot, \mathbf{0})$ has minimal fine limit 0 at $\mathbf{0}$ with respect to $\Omega_{\phi}$, and so $K_{\Omega_{\phi}}(t e, \mathbf{0}) / K_{\Omega_{\phi} \cup \mathbb{R}_{+}^{n}}(t e, \mathbf{0})$ has limit $\infty$ as $t \rightarrow 0+$. Thus, we conclude that $t^{n-1} K_{\Omega_{\phi}}(t e, \mathbf{0})$ has limit $\infty$ as $t \rightarrow 0+$.

Remark 5.5. For a non-Lipschitz function $\phi$, a relationship between the convergence of the integral $I^{+}$and minimal thinness of the difference $\Omega_{\phi} \backslash \overline{\mathbb{R}}_{+}^{n}$ was recently studied in $[\mathbf{1 1}]$. 
Acknowledgements. The author is very grateful to the referee for helpful comments and suggestions.

\section{Note added in proof.}

Recently, we have obtained a relationship between the boundary decay rate of the Green function and the growth rate of the Martin kernel (see [8]).

\section{References}

1. D. H. Armitage and S. J. Gardiner, Classical potential theory (Springer, 2001).

2. K. Burdzy, Brownian excursions and minimal thinness, III, Applications to the angular derivative problem, Math. Z. 192(1) (1986), 89-107.

3. K. Burdzy, Multidimensional Brownian excursions and potential theory, Pitman Research Notes in Mathematics, Volume 164 (Pitman, Boston, MA, 1987).

4. T. CARroll, A classical proof of Burdzy's theorem on the angular derivative, J. Lond. Math. Soc. (2) 38(3) (1988), 423-441.

5. T. CARroll, Boundary behaviour of positive harmonic functions on Lipschitz domains, Ann. Acad. Sci. Fenn. Math. 27(1) (2002), 231-236.

6. V. Eiderman and M. Essén, Harmonic majorization of $\left|x_{1}\right|$ in subsets of $\mathbb{R}^{n}, n \geqslant 2$, Ann. Acad. Sci. Fenn. Math. 21(1) (1996), 223-240.

7. S. J. Gardiner, A short proof of Burdzy's theorem on the angular derivative, Bull. Lond. Math. Soc. 23(6) (1991), 575-579.

8. K. Hirata, Estimates for the products of the Green function and the Martin kernel, Nagoya Math. J., in press.

9. R. A. Hunt and R. L. Wheeden, Positive harmonic functions on Lipschitz domains, Trans. Am. Math. Soc. 147 (1970), 507-527.

10. Ü. KurAn, On NTA-conical domains, J. Lond. Math. Soc. (2) 40(3) (1989), 467-475.

11. T. Lundh, Minimally thin sets below a function graph, Complex Variables 49 (2004), 639-645.

12. L. NAÏM, Sur le rôle de la frontière de R. S. Martin dans la théorie du potentiel, Annls Inst. Fourier 7 (1957), 183-281.

13. S. Sastry, Existence of angular derivative for a class of strip domains, Proc. Am. Math. Soc. 123(4) (1995), 1075-1082. 